\title{
EDITORIAL
}

\section{Cell phone use and ill health: Is there a definite relationship?}

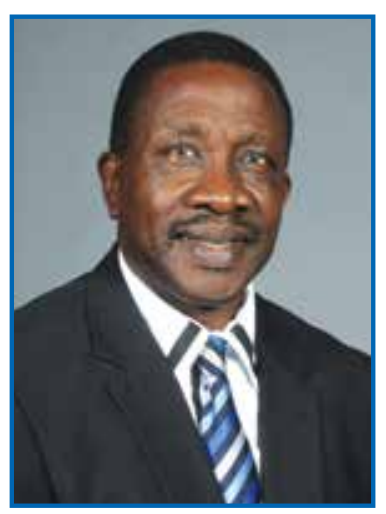

With the advent of the global use of cell phones, there have been concerns about their impact on the health of users. Interestingly, cell phone use is now an integral part of modern day communication between persons. In 2014, the World Health Organisation (WHO) estimated that there were 6.9 billion subscriptions globally and that in some parts of the world, mobile phones are the most reliable or the only phones available. ${ }^{1}$ Various studies have been conducted, although some are inconclusive on the link of cell phone use and ill health. Cell phones communicate by transmitting radio waves through a network of fixed antennas called base stations. Radiofrequency waves are electromagnetic fields, and unlike ionizing radiation such as X-rays or gamma rays, can neither break chemical bonds nor cause ionization in the human body. ${ }^{1}$

So what are the possible relationships between cell phone use and ill health? The operating low-powered radiofrequencies of cell phones are between 450 and $2700 \mathrm{MHz}$ with peak powers in the range of 0.1 to 2 watts. The radiofrequency exposure diminishes with increasing distance of the handset between the ear and hand and transmission power is only activated when the handset is switched on. Hence text messaging, Internet access, or use of a "hands free" device have a much lower exposure to radiofrequency fields than someone holding the handset against their head. ${ }^{1}$ However, the human body absorbs energy from devices that emit radiofrequency electromagnetic radiation such as cell phones, televisions, and radio transmissions.

One of the main concerns with cell phone use on health is the possibility of cancers due to the electromagnetic radiation. Despite "tissue heating" being the primary concern of electromagnetic radiation on the human body, it has been reported that at the frequencies of cell phones, the skin and other superficial tissues absorb most of the energy, resulting in negligible temperature rises in the brain or any other organs of the body. The WHO has documented that to date, research does not suggest any consistent evidence of adverse health effects from exposure to radiofrequency fields at levels below those that cause tissue heating. Further, research has not been able to provide support for a causal relationship between exposure to electromagnetic fields and self-reported symptoms, or "electromagnetic hypersensitivity". ' The latter is corroborated by the National Cancer Institute at the National Institutes of Health USA, which indicates that radiofrequency energy, unlike ionizing radiation, does not cause DNA damage that can lead to cancer. The only consistently observed biological effect in humans is tissue heating. ${ }^{2}$ In animal studies, it has not been found to cause cancer or to enhance the cancer-causing effects of known chemical carcinogens. ${ }^{3,4,5}$

However, cell phone use while driving is widely considered dangerous due to its potential for causing distracted driving and accidents. In the USA, a 2009 report documented 5474 people killed by distracted drivers. Of those, 995 were considered to have been killed by drivers distracted by cell phones. The report does not state whether this under- or overrepresents the level of cell phone use amongst drivers, and whether there is a causal relationship. ${ }^{6}$ The most current health concern is "Electromagnetic hypersensitivity" (EHS), which is human sensitivity to electromagnetic fields, to which negative symptoms have been attributed. EHS has no scientific basis and is not a recognised medical diagnosis. It is characterized by a "variety of non-specific symptoms, which include dermatological symptoms (redness, tingling, and burning sensations) as well as neurasthenic, and vegetative symptoms (fatigue, tiredness, concentration difficulties, dizziness, nausea, heart palpitation, and digestive disturbances). A survey of occupational medical centres estimated the prevalence of EHS to be a few individuals per million in the population. ${ }^{7}$ Treatment of EHS is symptomatic and includes establishing an effective physician-patient relationship to develop coping strategies with the situation and to encourage patients to return to work and lead a normal social life.

The move to the next generation of smart phones and $5 G$ cell phone technology aim at higher capacity than current $4 G$, allowing a higher density of mobile broadband users, and supporting device-to-device, ultra-reliable, and massive machine communications. The good news is that $5 \mathrm{G}$ research and development also aim at lower latency than $4 \mathrm{G}$ equipment and lower battery consumption, for better implementation. I hope that with the improvement in cell phone technology, more research will be conducted to conclusively confirm that its use is not associated or linked with ill-health. That is the essence of evidence-based research and medicine.

\section{Prof. Gboyega A Ogunbanjo}

Editor-in-chief: SAFPJ

\section{References:}

1. World Health Organisation (WHO). Electromagnetic fields and public health: mobile phones. Accessed on 7 Oct 2017. Available from: at http://www.who.int/mediacentre/factsheets/fs193/en/.

2. National Cancer Institute at the National Institutes of Health. Accessed on 7 Oct 2017. Available from: https://www.cancer.gov/about-cancer/causes-prevention/risk/radiation/cell-phones-fact-sheet

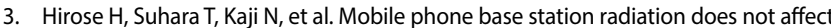
neoplastic transformation in BALB/3T3 cells. Bioelectromagnetics. 2008;29(1):55-64.

4. Oberto G, Rolfo K, Yu P, et al. Carcinogenicity study of $217 \mathrm{~Hz}$ pulsed $900 \mathrm{MHz}$ electromagnetic fields in Pim1 transgenic mice. Radiation Research. 2007;168(3):316-26.

5. Zook BC, Simmens SJ. The effects of pulsed $860 \mathrm{MHz}$ radiofrequency radiation on the promotion of neurogenic tumors in rats. Radiation Research. 2006;165(5):608-15.

6. US DOT National Highway Traffic Safety Administration Distracted Drive Report released September 2010. Accessed on 8 Oct 2017. Available from: http://www-nrd. nhtsa.dot.gov/Pubs/811379.pdf

7. World Health Organisation (WHO). Electromagnetic fields and public health Accessed on 9 Oct 2017. Available from: http://www.who.int/peh-emf/publications/facts/fs296/en/. 